\title{
Motor skill learning in the middle-aged: limited development of motor chunks and explicit sequence knowledge
}

\author{
Willem B. Verwey • Elger L. Abrahamse • \\ Marit F. L. Ruitenberg $•$ Luis Jiménez $•$ \\ Elian de Kleine
}

Received: 1 October 2010/ Accepted: 17 January 2011/Published online: 2 February 2011

(C) The Author(s) 2011. This article is published with open access at Springerlink.com

\begin{abstract}
The present study examined whether middleaged participants, like young adults, learn movement patterns by preparing and executing integrated sequence representations (i.e., motor chunks) that eliminate the need for external guidance of individual movements. Twentyfour middle-aged participants (aged 55-62) practiced two fixed key press sequences, one including three and one including six key presses in the discrete sequence production task. Their performance was compared with that of 24 young adults (aged 18-28). In the middle-aged participants motor chunks as well as explicit sequence knowledge appeared to be less developed than in the young adults. This held especially with respect to the unstructured 6-key sequences in which most middle-aged did not develop independence of the key-specific stimuli and learning seems to have been based on associative learning. These results are in line with the notion that sequence learning involves several mechanisms and that aging affects the relative contribution of these mechanisms.
\end{abstract}

\section{Introduction}

The impact of aging on society is of great concern. The $60+$ population across Europe is expected to rise from $20 \%$

W. B. Verwey $(\bowtie) \cdot$ E. L. Abrahamse .

M. F. L. Ruitenberg - E. de Kleine

Cognitive Psychology and Ergonomics, University of Twente, P.O. Box 217, 7500 AE Enschede, The Netherlands

e-mail: w.b.verwey@utwente.nl

L. Jiménez

University of Santiago, Santiago de Compostela, Spain today to about 35\% in 2050 (e.g., Rynning, 2008). Consequently, there is a debate in many countries whether the age of retirement should increase. In the Netherlands and Spain, for example, the retirement age will rise in the coming years from 65 to 67 . This implies that in the near future the working population will include more people at advanced ages. These people too will have to learn new skills. It is known, however, that many cognitive functions decline with age (e.g., Perry, McDonald, Hagler et al., 2009; Rabbitt, 1997; Seidler, Bernard, Burutolu et al., 2010). In fact, cognitive decline appears to accelerate after the age of 50 but the onset can be observed already before that age (Verhaeghen \& Salthouse, 1997). As cognition is important for developing new motor skills (e.g., Seidler et al., 2010), in the present study, we explore the extent to which people that are going toward the end of their professional career are still able to develop new motor skills. At a practical level, this study is aimed at unveiling problems of this age group in acquiring motor skills, and at providing indications as to how the training for this particular group can be improved (Seidler, 2007). At a theoretical level, the present study is important because aging may have different effects on the mechanisms underlying the acquisition of motor skills in young adults, and may therefore contribute to understand when certain mechanisms are being used.

Several researchers have argued that, apart from being generally slower, older people are as capable as young adults in acquiring new skills. For example, Durkin, Prescott, Furchtgott, Cantor and Powell (1995) found similar improvements on a pursuit rotor task across ages and argued that acquisition of non-declarative tasks may not be affected by age. In a similar vein, Howard and Howard (1992) and Salthouse, McGuthry, and Hambrick (1999) argued that motor sequence learning in a serial response 
time task is not affected by age. Daselaar, Rombouts, Veltman, Raaijmakers, and Jonker (2003) stated that "age differences in motor skill learning, if any, are subtle" (p. 1014), and Voelcker-Rehage (2008) concluded from a review of the literature that learning capabilities remain intact in older adults. However, this notion contrasts with anecdotic indications that high skills in sports and playing music require practice at younger ages suggesting that it is much more difficult to acquire such skills later in life. Indeed, evidence is mounting that the development and use of complex motor skills do decline with age not only for biomechanical and neuromuscular reasons but also due to cognitive decline (Seidler et al., 2010). For example, motor tasks may well be controlled in a qualitatively different and less efficient way in that execution requires more cognitive control and external guidance (Hedel \& Dietz, 2004; Li \& Lindenberger, 2002; Rabbitt, 1997).

Research with young adults has indicated that the development of sequential motor skills involves the creation of integrated motor representations in memory called motor chunks. These motor chunks can be considered building blocks of skilled behavior because they allow the rapid and automatic execution of fixed movement patterns while reducing the need for cognitive and external control (e.g., Gallistel, 1980; Rhodes, Bullock, Verwey, Averbeck, \& Page, 2004; Shea, Park \& Wilde Braden, 2006; Paillard, 1960; Verwey, 1999; for a further subdivision of motor chunks, see De Kleine \& Verwey, 2009). It is likely that such integrated movement patterns are at the basis of professional activities like controlling factory systems and vehicles, and of sports activities like serving in tennis. In the absence of these integrated movement patterns such tasks would be highly attention and time demanding because an explicit decision has to be made about each individual movement. The generality of the chunking mechanism for motor skills is demonstrated by indications for chunk-based motor control in different sequential movement tasks like moving a lever to sequentially presented targets using elbow flexions and extensions (e.g., Park and Shea, 2005; Panzer, Krueger, Muehlbauer, Kovacs, \& Shea, 2009), moving a pen through a cut-out maze pattern with the eyes closed (e.g., van Mier \& Hulstijn, 1993; van Mier \& Petersen, 2006), uttering speech (e.g., Bohlanda \& Guenther, 2006), and pressing discrete and fixed series of keys (e.g., Bo \& Seidler, 2009; Sakai, Kitaguchi, \& Hikosaka, 2003; Verwey, 1999; Verwey, Abrahamse, \& Jiménez, 2009).

\section{The discrete sequence production task}

To explore motor chunking in middle-aged people we used the discrete sequence production (DSP) task. This task involves the sequential display of typically three to six stimuli each requiring a key press. Each next stimulus is displayed immediately after the response to the previous stimulus has been given. This set up yields a series of response times (RTs). ${ }^{1}$ In a DSP experiment participants usually practice two such key press sequences that are administered in a random order. Given the fixed order of the responses in each of the DSP sequences, young adults have been found to gradually switch from reacting to each key-specific stimulus in the reaction mode to executing series of key presses as a whole in the sequencing mode. In the sequencing mode they select and execute one or more existing motor chunks (Rhodes et al., 2004; Verwey, Lammens, \& van Honk, 2002). Therefore, in the typical DSP task young adults change with practice from executing two fixed series of three to six 6-choice RT tasks (in case of using 6 fingers), to executing a single 2-choice reaction time task in which an entire keying sequence is produced in response to the first stimulus. The DSP task is attractive for understanding sequential movement control because the fast development of the sequencing skill and the rapid execution of individual and simple movements in this task allow for tracing the control processes underlying the control of movement sequences (e.g., MacKay, 1982; Rhodes et al., 2004).

The use of motor chunks in a familiar DSP sequence is indicated by: (a) the rapid execution of the individual key presses past the first with RTs sometimes below $100 \mathrm{~ms}$ (Verwey, 1999), (b) the possibility to execute these sequences without key-specific stimuli past the first (Verwey, 1999, 2010), (c) the continued use of the timing pattern developed during practice (Verwey, 1996; Verwey et al., 2009), and (d) the limited effect of a secondary task on sequence execution (Verwey, Abrahamse, \& De Kleine, 2010). Research showed that selection of a familiar keying sequence can occur during execution of a preceding familiar sequence and selection demands do not affect ongoing execution (Verwey, 1995, 2001). These and other findings led to the dual processor model for discrete keying sequences (Verwey, 2001). This model asserts that the motor chunk is selected and loaded into the motor buffer by a cognitive processor. Then the motor processor reads each element from the motor buffer and immediately executes it. As execution does not require the cognitive processor this model explains also the reduced attentional demands of executing familiar sequences (e.g., Verwey, 2003b; for an extension of the model, see Verwey et al., 2010).

\footnotetext{
${ }^{1}$ With respect to the DSP task, the name 'response time' is preferred over 'reaction time' because with practice individual key presses are no longer reactions to stimuli.
} 


\section{The previous study on elderly}

Recently, Verwey (2010) compared performance of elderly over 74 and young adults on 3- and 6-key DSP sequences. The results corroborated that elderly are slower while in terms of learning curves they seemed to confirm that elderly and young adults have similar learning capacities (e.g., Voelcker-Rehage, 2008). However, more detailed analyses revealed an important age difference: While in the ensuing test phase young adults showed evidence for sequence-specific learning in that they were much faster on familiar than on unfamiliar sequences, this was hardly the case with the elderly. In line with earlier research (Shea et al., 2006) the RT patterns were not typical for the use of motor chunks, and in contrast to most young adults the elderly were not able to execute the sequences when only the first stimulus was presented. These indications that motor chunks had not been developed by the elderly corroborated earlier findings that elderly are more dependent on external guidance than young people (e.g., Hedel \& Dietz, 2004; Hultsch, Hertzog, \& Dixon, 1987).

Interestingly, the elderly did show some sequence-specific learning in that there was a relatively small advantage of the familiar over the unfamiliar 6-key sequence that gradually increased with sequential position. Given that elderly continued to respond to key-specific stimuli and the benefit of practice increased toward the end of the unstructured 6-key sequence these data were taken as an indication that the sequence-specific improvement was due to associative learning (cf. MacKay, 1982; Spiegel \& MacLaren, 2006). In the serial RT task-in which participants respond to successive key-specific stimuli tooassociative learning is assumed to occur at several processing levels in (e.g., Abrahamse, Jiménez, Verwey, \& Clegg, 2010; Keele, Ivry, Hazeltine, Mayr, \& Heuer, 2003). The results of the DSP study with elderly (Verwey, 2010) are in line with this notion of associations between successively used representations at various processing levels. That is, if associations at the motor level are dominant, we say that a motor chunk has developed and response-specific stimuli are no longer required. In that case, advance preparation of the motor chunk seems to be required which may require some degree of explicit (i.e., verbalizable) knowledge (Verwey, 2003a). If for some reason motor chunks do not develop or are not prepared, the development of associations at some earlier processing level (e.g. between successive stimuli) may improve performance. Then, stimuli continue to be used in the successive choice response tasks but a familiar order speeds up the processing of the individual stimuli.

There is indeed some evidence that motor chunking (requiring preparation and involving the ignoring of key- specific stimuli) and associative learning (involving continued responding) are distinct learning mechanisms. That is, skill in discrete keying (DSP) sequences did not transfer to the serial RT task (Verwey, 2003a). Also, no evidence could be found that motor chunks are responsible for improvement in the serial RT task (Jiménez, 2008; Jiménez, Méndez, Pasquali, Abrahamse, \& Verwey, submitted; but see Koch \& Hoffmann, 2000 for an exception with a highly structured serial RT sequence). In line with associative learning in elderly performing the DSP task (Verwey, 2010), several serial RT studies found limited or no deterioration of sequential learning with age (e.g., Howard, Howard, Dennis, Yankovich \& Vaidya, 2004).

\section{The present study on middle-aged participants}

To explore the capacity to learn new motor skills of people who are nearing their retirement we examined the performance of participants between 55 and 62 executing fixed 3and 6-key sequences. For simplicity these participants will here be called middle-aged though we are aware that this name is typically used for younger people too (from say 40 to 65 years of age). We anticipated that these middle-aged would use motor chunks for short series of key presses because, first, Verwey (2010) established that even elderly up till 80 use some rudimentary form of chunking for 3-key sequences in a DSP task. Also, Bo, Borza and Seidler (2009) found indications in a 12-item sequential keying task that many of their older participants (aged 65-78) spontaneously used 3-key chunks (though these chunks were shorter than those of young adults who used 4-key chunks). Given this capacity of older people to develop at least short motor chunks we further anticipated that the middle-aged would benefit from dividing longer sequences into 3-key segments (Shea et al., 2006; cf. Park, Wilde, \& Shea, 2004; Wightman \& Lintern, 1985). This was examined by having half the participants practice the 6-key sequence with a pause halfway through the sequence. This so-called prestructured group practiced a sequence that was divided into two 3-key segments. The remaining unstructured participants practiced the 6-key sequence without such a pause.

Practice was followed by a test phase that (like in Verwey, 2010) included three conditions: a familiar condition in which participants carried out the practiced 3- and 6-key sequences in response to the key-specific stimuli (but without the pause), a single-stimulus condition in which the same familiar sequences were to be produced in response to just the first stimulus, and the unfamiliar condition in which a new 3- and a new 6-key sequence were to be generated in response to key-specific stimuli. 
We distinguished between indicators for general learning, for sequence-specific learning, and for three mechanisms that may underlie sequencing skill (i.e., using motor chunks, associative learning, and explicit knowledge). These indicators were then used to hypothesize differences between the middle-aged and the young adults. General (i.e., sequence-unspecific plus sequence-specific) learning is assumed to be indicated by (a1) a reduction of total sequence execution time in the practice phase, and by (a2) an increasing difference between sequence initiation time and mean interkey interval. Our first hypothesis was that middle-aged would be slower than the young adults (i.e., Hypothesis 1a), and that - given the earlier studies-both age groups would show similar improvement with practice (Hypothesis 1b). It was not clear whether the difference between sequence initiation time and mean interkey interval would distinguish between the age groups.

Sequence-specific learning is indicated by (b1) faster execution of familiar than of unfamiliar sequences in the test phase. Because responses past the first are probably primed more in familiar than unfamiliar sequences by preceding responses (i.e., by a build up of activation) we also assumed that sequence-specific learning would be reflected in (b2) a larger difference between the time to initiate the sequence (i.e., $T_{1}$ ) and the mean of the ensuing interkey intervals (IKIs) in the familiar sequences than in the unfamiliar sequences ${ }^{2}$ (cf. Verwey, 2010). If performance of the middle-aged is in between that of the young adults and of the elderly in Verwey (2010), the second hypothesis was that sequence-specific learning would be less in the middle-aged than in the young adults.

Sequence-specific learning based on motor chunks is assumed to be indicated by (c1) little performance reduction when key-specific stimuli past the first are not presented. Also, there should be (c2) a rapid RT decrease after the first response of each motor chunk (inducing a discontinuity at the second response), and (c3) a relatively slow response where the pause in the prestructured sequence induced a transition between successive motor chunks during practice. ${ }^{3}$ In contrast, associative sequencespecific learning implies that key-specific stimuli continue to be used causing (d1) very limited capacity to execute the familiar sequences when key-specific stimuli past the first are not presented, and (d2) execution rate to be slower than when motor chunks are used. Importantly, (d3) the

\footnotetext{
${ }^{2}$ In Verwey $(2010)$ the $T_{1}$ versus IKI difference in the familiar relative to the unfamiliar sequence was presented as chunking index. Given that this index is sensitive to associative learning too, the $T_{1}$ versus IKI difference in familiar relative to unfamiliar sequences is used here to indicate sequence-specific learning overall.

3 Attempts failed to demonstrate that such a slow response is caused by the development of a rhythm (Verwey, 1996; Verwey \& Dronkert, 1996; Verwey et al., 2009).
}

advantage of familiar over unfamiliar sequences should gradually increase with sequence position (MacKay, 1982; Verwey, 2010). The third hypothesis, then, was that the middle-aged develop motor chunks for the 3-key segments of the 3-key and the prestructured 6-key sequence. It was not clear at the outset whether the unstructured 6-key sequence would involve the use of motor chunks (like in young adults) or whether indications for associative learning would be observed (like in the elderly).

Participants in DSP task studies have been found to also develop explicit, verbalizable sequence knowledge (e.g., Verwey, 2003a, 2003b; Verwey \& Eikelboom, 2003). The relatively broad and abstract character of explicit knowledge probably allows it to be accessed and used flexibly by various cognitive processes (e.g., Baars, 1997). Especially in the single-stimulus condition, explicit knowledge is likely to be used for sequence execution but the required translation processes for each key press would considerably slow execution (Cleeremans \& Sarrazin, 2007). As explicit knowledge-more than implicit, nonverbalizable sequence knowledge-is assumed to be flexible in its application we assumed that availability of explicit knowledge can best be tested by examining awareness of the sequences in a way that is new to the participants. We assumed that more aware participants would (e1) be better able to write down the order of the keys in a recall test, and (e2) would better be able to select their sequences from a set of alternatives (e.g., Verwey \& Eikelboom, 2003). Earlier research indicates that aging reduces the capacity to develop explicit sequence knowledge (e.g., Gaillard et al., 2009; Prull, Gabrieli, \& Bunge, 2000; Verwey, 2010), so explicit awareness was expected to be more limited in the middleaged than in young adults (also reducing performance in the single-stimulus condition). The fourth hypothesis was that middle-aged participants would develop less awareness of their sequences, and that aware participants would primarily use their explicit knowledge for sequence execution in the single-stimulus condition (especially with 3-key sequences).

\section{Method}

\section{Participants}

The experiment involved 24 middle-aged participants between 55 and 62 years (mean age 58, 14 women). Before starting the experiment participants filled out an informed consent form. One of the middle-aged participants was replaced because she had too many response errors (18\% of all her sequences had an error as opposed to the others $<12 \%$ ). Like in the Verwey (2010) study, participants were recruited by the three students carrying out the experiment 
and often constituted of their relatives. Care was taken that participants were always tested by a student who was not a relative.

The middle-aged participants were screened with a questionnaire on health problems that might influence participants' performance. It showed that 18 of the 24 middle-aged participants were entirely healthy and used no medication. One of the participants indicated to suffer from arthritis, but after the experiment she indicated to have had no problems executing the keying sequences. Health problems mentioned most by the remaining five participants, concerned hypertension which was treated with hypertension medication. The study had been approved by the ethics committee of the University of Twente.

The results of these middle-aged participants were compared with those of the 24 young adults (mean age 22 , range $18-28,16$ women) who had also been used as a control group in the Verwey (2010) study.

\section{Tasks}

The task was identical to the one reported in Verwey (2010). Six black $11 \times 11 \mathrm{~mm}$ placeholders were displayed on a laptop computer display at a $12-\mathrm{mm}$ mutual distance against a white background. Between the third and fourth placeholder was a 37-mm gap with the letter ' $\mathrm{H}$ ' in the middle to mimic placement of the (DFG and JKL) keys at the keyboard on both sides of the $\mathrm{H}$ key. As soon as a placeholder was filled with green, participants pressed the associated key with their left or right ring, middle, or index finger. When the correct key had been pressed, the color in the square changed back to the white background color.

Each participant responded to stimuli presented in two fixed orders (i.e., $S_{1}-S_{6}$ and $S_{1}-S_{3}$ ), yielding responses in a fixed 6-key sequence $\left(R_{1}-R_{6}\right)$ and in a fixed 3-key sequence $\left(R_{1}-R_{3}\right)$. The RT between Stimulus $\mathrm{n}$ and Response $\mathrm{n}$ is indicated by $T_{\mathrm{n}}$ (e.g., the RT between $S_{2}$ and $R_{2}$ is $T_{2}$ ). As the response stimulus interval (RSI) was zero RTs past the first can be regarded interkey intervals (IKIs). The term trial is used to denote an entire keying sequence.

Following a sequence the display was erased white for $2,000 \mathrm{~ms}$ to indicate completion of the sequence. Then the black outlines were presented again for 1,000 ms and the first stimulus of the next sequence was displayed. Pressing a false key resulted in an error message for $500 \mathrm{~ms}$. The ongoing sequence was then aborted, and followed by a $1,000 \mathrm{~ms}$ empty screen after which the next sequence started with the $1,000 \mathrm{~ms}$ presentation of empty squares. In the practice phase half the participants in each age group (i.e., the prestructured group) had a pause between $R_{3}$ and $S_{4}$. The pause imposed a segmentation structure onto this sequence (e.g., Verwey, 1996). As fixed RSIs might induce learning of a fixed rhythm and therewith prevent development of two motor chunks, we decided to use a nonaging interval of at least $300 \mathrm{~ms}$. Intervals are non-aging when the probability that the RSI ends reduces with RSI length. This prevents the participant's expectancy for the stimulus to rise as the RSI lasts longer (Gottsdanker, Perkins, \& Aftab, 1986). We computed the non-aging RSI in a programming loop in which each time $5 \mathrm{~ms}$ was added. After each addition there was a $1 \%$ chance that the RSI stopped; otherwise another $5 \mathrm{~ms}$ was added until total duration amounted to $2,000 \mathrm{~ms}$. The remaining participants without a pause in their 6-key sequence made up the unstructured group.

Across all participants, keys (and therewith fingers) were counterbalanced across sequential positions so that each of the six fingers contributed as much to each RT at a particular sequential position. For example, one participant in the unstructured sequence group had KFGDJL and FKL, the next participant had LGJFKD and GLD, and so on. Similarly, in the prestructured group one participant had KFG-DJL and FKL while the next participant had LGJFKD and GLD ('-' indicating the pause). In each block of trials, the 3- and 6-key sequences were carried out in random order.

Each practice block included 24 6-key and 24 3-key sequences that were presented in random order. With 6 practice blocks this yielded a total of 144 practice trials for each sequence. Halfway through each practice block there was a $40 \mathrm{~s}$ resting period and each practice block was followed by a rest period of at least $4 \mathrm{~min}$. Each practice block was followed by a display of the percentage of errors and mean RT.

The test phase included three blocks of trials, each with a different experimental condition separated by a $40 \mathrm{~s}$ pause and including repeated execution of a 3- and a 6-key sequence in random order. In each test block each of the two sequences was presented 12 times in random order. The order of the three test blocks was counterbalanced across participants. Two test blocks contained the two familiar sequences. Of these two, one included the same key-specific stimuli as the practice phase (i.e., the familiar condition), whereas the other block presented just the first key-specific stimulus after which each key press was followed by lighting up of all placeholders simultaneously (single-stimulus condition): Each time a key was pressed, all placeholders briefly became white and were then filled with green again. This required participants to complete the sequence without external guidance. The third test block contained a 3-key and a 6-key sequence that were new to the participant (unfamiliar condition). Due to the balancing procedure these unfamiliar sequences were familiar to other participants. So, across all participants the same set of 
six 3- and six 6-key sequences was used as familiar and unfamiliar sequences. The test phase did not include pauses during any of the 6-key sequences. Like in the practice phase, the interval between depressing the last key of one sequence and presenting the first key-specific stimulus of the next sequence in the test phase amounted to 3,000 ms. Each test block was followed, again, by displaying the percentage of errors and mean RT.

\section{Procedure}

The procedure was identical to that used in Verwey (2010) with elderly and young adults in that the middleaged participants were visited at home upon appointment by an experimenter. Before starting, the experimenters tried to preclude any interruptions during the experiment (e.g., by turning off the telephone). Participants started by filling out an informed consent form. An ad hoc questionnaire was used to assess potential health problems, use of prescribed medication, problems with concentration, possibility of using all ten fingers, educational level, regular computer use, and experience with typing, computers and playing piano. Finally, participants received a written instruction on the task to be performed. This was extended orally by the experimenter.

Next, participants performed 6 practice blocks. Following the practice phase, awareness of the sequences was tested with a questionnaire. Participants were asked to write down as accurately as possible the two practiced sequences by using the letters of the keys employed in the experiment ('recall' test). As a reminder, the positions of the 6 keys on the keyboard were printed in the questionnaire in their normal configuration next to each other with the ' $\mathrm{H}$ ' marked in the center position. Also, the laptop keyboard remained in sight for consultation. Next, participants were asked to select their 6-key sequence from a set of 12 alternatives, and their 3-key sequence from another set of 12 ('recognition' test).

Following this questionnaire, participants performed the test phase. In the single-stimulus condition participants were encouraged to use their gut feeling when they were not sure which key to press next. Again, pressing a false key resulted in displaying an error message and aborting the sequence, after which the next sequence started. For each participant the experiment lasted 1.5-2 h.

\section{Apparatus}

Stimulus presentation, timing, and data collection was achieved using the E-prime ${ }^{\complement} 2.0$ experimental software package on a standard Pentium ${ }^{\circledR}$ IV notebook computer (Sony Vaio PCG-Z1SP) in which all unnecessary Windows
XP services had been switched off to allow accurate time measurement. Stimuli were presented on the $232 \times$ $138 \mathrm{~mm}\left(10.6^{\prime \prime}\right)$ notebook display running at 1,280 by 768 pixel resolution in 32-bit color. The keys on the regular key computer board measured $16 \times 16 \mathrm{~mm}$ with a $1 \mathrm{~mm}$ space between adjacent keys. The viewing distance usually is approximately $50 \mathrm{~cm}$ with this type of computer but this was not controlled.

Design and analyses

Mean RTs per key, sequence, participant and phase were submitted to ANOVAs. All key presses of sequences with one or more errors were omitted from RT analyses. The practice and test phases involved separate RT ANOVAs for 3- and 6-key sequences, though in the single-stimulus condition of the test phase the variable of main interest was the number of correctly produced sequences.

Effect sizes are reported in terms of partial eta-squared $\left(\eta_{\mathrm{p}}^{2}\right)$. Planned comparisons were used to test the hypotheses presented in the "Introduction". Individual sequences were omitted from the analyses when total execution time was longer than the mean across participants in a certain age group plus 2.5 times the standard deviation. This was done separately across Blocks 1 and 2 (thresholds for young and middle-aged: 600 and 1,200 ms), and Blocks 3-6 (500 and 1,000 ms). This led to exclusion of $2.3 \%$ of all sequences in the practice phase. In the test phase, thresholds were computed separately for the familiar and unfamiliar sequence conditions, and separately for middle-aged and young participants. Across these two conditions thresholds were 550 and $650 \mathrm{~ms}$ for young adults and 1,050 and 1,050 $\mathrm{ms}$ for the middle-aged. This yielded removal of $2.7 \%$ of the test phase sequences from the reaction time analyses. Proportions of errors were transformed with an arcsine function before being subjected to variance analytic analyses (Winer, Brown, \& Michels, 1991).

\section{Results}

Practice phase

\section{3-Key sequences}

Individual RTs of the 3-key sequence were analyzed with a mixed 2 (Age) $\times 6$ (Block) $\times 3$ (Key) design with Age as between-subjects variable. Main effects showed that, as expected, mean RT reduced with Block, $F(5,230)=$ 157.2, $p<0.001, \eta_{\mathrm{p}}^{2}=0.77$, and the middle-aged participants were considerably slower than the young adults, 531 versus $284 \mathrm{~ms}, F(1,46)=81.9, p<0.001, \eta_{\mathrm{p}}^{2}=0.64$. In 


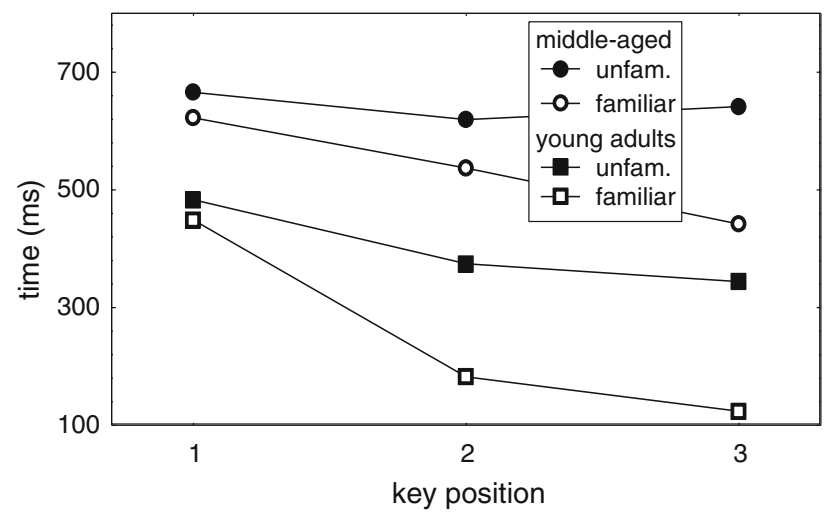

Fig. 1 Response times in the familiar and unfamiliar 3-key sequences of the test phase as a function of key position and age group

line with the notion of a similar general learning capacity in both age groups, the absence of a significant Age $\times$ Block interaction suggested that the improvement over practice was not different for both age groups, $F(5$, 230) $=0.5, p>0.20, \eta_{\mathrm{p}}^{2}=0.01$. (i.e., Indicator a1 supports Hypothesis 1a).

The Key main effect, $F(2,92)=54.1, p<0.001$, $\eta_{\mathrm{p}}^{2}=0.54$ (cf. test phase results in Fig. 1) showed that RT reduced with Key location. According to an Age $\times$ Key interaction this reduction was larger for the young adults than for the middle-aged, $F(2,92)=13.6, p<0.001$, $\eta_{\mathrm{p}}^{2}=0.23$. The Age $\times$ Block $\times$ Key interaction, $F(10$, $460)=6.9, p<0.001, \eta_{\mathrm{p}}^{2}=0.13$, indicated that $T_{2}$ and $T_{3}$ reduced more with practice than $T_{1}$, and that this reduction was smaller for the middle-aged than for the young adults. Planned comparisons confirmed that in both age groups there was a larger reduction across Blocks $1-6$ of $T_{2}$ and $T_{3}$ than of $T_{1}$ : For the middle-aged the $T_{1}$ versus $T_{2} T_{3}$ difference increased from $-18 \mathrm{~ms}$ in Block 1-94 ms in Block $6, F(5,230)=13.9, p<0.001, \eta_{\mathrm{p}}^{2}=0.23$. For the young adults the $T_{1}$ versus $T_{2} T_{3}$ difference increased from $24 \mathrm{~ms}$ in Block 1-239 $\mathrm{ms}$ in Block $6, F(5,230)=58.8$, $p<0.001, \eta_{\mathrm{p}}^{2}=0.56$, and the $T_{1}$ versus $T_{2} T_{3}$ increase was larger for the young adults, $F(5,230)=8.6, p<0.001$, $\eta_{\mathrm{p}}^{2}=0.16$. (i.e., Indicator a 2 rejects Hypothesis $1 \mathrm{~b}$ ). So, total execution time suggested a similar improvement across age groups, but the $T_{1}$ versus $T_{2} T_{3}$ difference indicated that the middle-aged learned less than the young adults.

\section{6-Key sequences}

The 6-key version of the above ANOVA also included the between-subject Structure variable (distinguishing prestructured and unstructured sequences). It showed a substantial Age effect too, 523 versus $283 \mathrm{~ms}, F(1,44)=$ 69.1, $p<0.001, \eta_{\mathrm{p}}^{2}=0.61$ (i.e., Indicator a1 supports
Hypothesis 1a). Improvement across Blocks 1-6 was significant, $F(5,220)=227.1, \quad p<0.001, \quad \eta_{\mathrm{p}}^{2}=0.84$, also when tested for each age group separately, $F_{\mathrm{s}}(5$, $220)>110.9, \quad p s<0.001, \quad \eta_{\mathrm{p}}^{2} \mathrm{~s}>0.72$. Again, nonsignificance of the Age $\times$ Block interaction suggested similar improvement in both age groups, $F(5,220)=$ $0.24, p>0.20, \eta_{\mathrm{p}}^{2}=0.01$. (i.e., Indicator a1 supports Hypothesis 1b).

The effect of Key, $F(5,220)=28.9, \quad p<0.001$, $\eta_{\mathrm{p}}^{2}=0.40, \quad$ interacted with Age, $F(5,220)=7.6$, $p<0.001, \eta_{\mathrm{p}}^{2}=0.15$, and Block, $F(25,1,100)=20.2$, $p<0.001, \eta_{\mathrm{p}}^{2}=0.31$. An additional Age $\times$ Block $\times$ Key interaction, $F(25,1,100)=2.6, p<0.001, \eta_{\mathrm{p}}^{2}=0.06$, suggested that the difference between $T_{1}$ and the mean of $T_{2}-T_{6}$ increased less with practice in the middle-aged than in the young adults (this was significant also when $T_{4}$ was excluded). Planned comparisons corroborated this suggestion as they showed that the difference between $T_{1}$ versus $T_{2} T_{3} T_{5} T_{6}$ increased with practice for each group, $F_{\mathrm{s}}(5,220)>20.9, p \mathrm{~s}<0.001, \eta_{\mathrm{p}}^{2} \mathrm{~s}>0.32$, and that this difference increased less with practice for middle-aged than for young adults, $F(5,220)=5.5, p<0.001$, $\eta_{\mathrm{p}}^{2}=0.11\left(T_{1}\right.$ vs. $T_{2} T_{3} T_{5} T_{6}$ difference for middle-aged: from $-19 \mathrm{~ms}$ in Block 1-101 $\mathrm{ms}$ in Block 6, for young adults: from $49 \mathrm{~ms}$ to $258 \mathrm{~ms}$ ). (i.e., Indicator a2 rejects Hypothesis 1b). So, like with the 3-key sequences mean response time suggested a similar improvement across age groups, but the difference between $T_{1}$ and mean IKI increased faster with practice for the young adults than for the middle-aged.

In hindsight we wondered whether-given the generally slower responses in the middle-aged-improvement in the practice phase could perhaps have been better explored with RTs standardized within each age group to compensate for the absolute differences in response speed (Faust, Balota, Spieler, \& Ferrarro, 1999). Performing such analyses confirmed that in relative terms the $\mathrm{RT}$ reduction in the 3-key and the unstructured 6-key sequence was less in the middle-aged than in the young adults. Specifically, repeating the practice phase ANOVAs for the 3-key sequence with standardized RTs showed that from Block 1-6 the middle-aged improved only $75 \%$ of the improvement observed with the young adults, $F(5,230)=2.7$, $p<0.05, \eta_{\mathrm{p}}^{2}=0.06$. For the 6-key sequence, improvement of the middle-aged was smaller than of young adults too, $F(5,220)=2.7, \quad p<0.05, \quad \eta_{\mathrm{p}}^{2}=0.06$ : This time, an Age $\times$ Block $\times$ Structure interaction, $F(5,220)=3.2$, $p<0.01, \eta_{\mathrm{p}}^{2}=0.07$, showed that improvement of the middle-aged from Block 1-6 was only $62 \%$ of that of the young adults in the unstructured 6-key sequence, $F(5$, $220)=5.6, p<0.001, \eta_{\mathrm{p}}^{2}=0.11$. However, it was as large in both age groups in the prestructured 6-key sequence, $F(5,220)=0.3, p>0.20, \eta_{\mathrm{p}}^{2}=0.01$. (i.e., 
Indicator a1 now rejects Hypothesis 1b). These findings confirm that the apparent similarity in learning rate of older people in several earlier studies (see Voelcker-Rehage, 2008) may have been caused by ignoring the baseline differences, and that standardized RTs may be preferred when comparing groups with different baselines.

In summary, the practice phase confirms that the middle-aged were slower (supporting Hypothesis 1a) and that improvement in the age groups for total execution time was similar (supporting Hypothesis 1b). However, less improvement for the middle-aged is observed when examining the $T_{1}$ versus IKI difference and standardized RTs (rejecting Hypothesis 1b).

\section{Errors}

Arcsine transformed error proportions of 3- and 6-key sequences were submitted also to mixed 2 (Age) $\times 6$ (Block) $\times 3$ (Key) and 2 (Age) $\times 6$ (Block) $\times 2$ (Structure) $\times 6$ (Key) ANOVAs. In the 3-key sequence, error proportions were not different for the two age groups: $F(1$, $44)=0.0, p>0.20, \eta_{\mathrm{p}}^{2}=0.00$. Average error rate per key amounted to $1.2 \%$ (error rates are relatively low because sequence execution stopped once an error had been made). Furthermore, in the 3-key sequence, participants made most errors on the second key $(0.6,2.1,1.0 \%$, resp.), $F(2$, $88)=11.9, p<0.001, \eta_{\mathrm{p}}^{2}=0.21$. In the 6-key sequence, middle-aged participants made somewhat fewer errors than young adults: $F(1,44)=4.1, p<0.05, \eta_{\mathrm{p}}^{2}=0.09$ (1.0 vs. $1.4 \%$ per key). The prestructured group made fewer errors than the unstructured group ( 0.9 vs. $1.5 \%$ per key), $F(1$, 44) $=7.6, p<0.01, \eta_{\mathrm{p}}^{2}=0.15$.

\section{Test phase}

\section{Familiar and unfamiliar sequences}

3-Key sequences. Figure 1 suggests that in both pre- and unstructured sequences the middle-aged were slower than the young adults. They also seemed to have a smaller $T_{1}$ versus $T_{2} T_{3}$ difference than young adults and did not show the discontinuity at $R_{2}$ that we consider typical for motor chunk use. RTs of the 3-key sequences were analyzed with a mixed 2 (Age) $\times 2$ (Familiarity: familiar vs. unfamiliar sequence) $\times 3$ (Key) ANOVA. It confirmed the expected age difference on overall RTs (588 vs. $326 \mathrm{~ms}), \quad F(1,46)=99.4, \quad p<0.001, \quad \eta_{\mathrm{p}}^{2}=0.68$. As expected, sequence-specific learning was indicated by the fact that the familiar 3-key sequence was executed faster than the unfamiliar 3-key sequence, $F(1,46)=99.0$, $p<0.001, \eta_{\mathrm{p}}^{2}=0.68$ (Indicator b1). Planned comparisons showed that the advantage of the familiar over the unfamiliar sequence was significant for both age groups separately, $F \mathrm{~s}(1,46)>35.2, p \mathrm{~s}<0.001, \eta_{\mathrm{p}}^{2}=0.43$. The Age $\times$ Familiarity interaction was not significant, $F(1$, 46) $=2.44, p>0.12, \eta_{\mathrm{p}}^{2}=0.05$, suggesting that the extent of sequence-specific learning of the 3-key sequences was not different between both age groups (Indicator b1 rejects Hypothesis 2).

The Key main effect indicated that $T_{1}$ was generally longer than $T_{2}$ and $T_{3}, F(2,92)=65.2, p<0.001$, $\eta_{\mathrm{p}}^{2}=0.59$. The Familiarity $\times$ Key interaction, $F(2$, $92)=19.1, p<0.001, \eta_{\mathrm{p}}^{2}=0.29$, was in line with the notion that the $T_{1}$ versus $T_{2} T_{3}$ difference was larger for familiar than for unfamiliar 3-key sequences. The Age $\times$ Key interaction, $F(2,92)=11.3, p<0.001$, $\eta_{\mathrm{p}}^{2}=0.20$, suggested that the $T_{1}$ versus $T_{2} T_{3}$ difference was larger for young than for middle-aged participants. The notion that the RT difference between the three responses would differ for the familiar and unfamiliar sequences, and that this difference would in turn be different for the two age groups, was in line with a marginally significant Age $\times$ Familiarity $\times$ Key interaction, $F(2,92)=2.5$, $p<0.09, \eta_{\mathrm{p}}^{2}=0.05$. Planned comparisons addressed these indications more specifically. A first planned comparison confirmed that for the middle-aged, $T_{2}$ and $T_{3}$ together were shorter than $T_{1}$ for the familiar sequence, $F(1$, 46) $=21.1, p<0.001, \eta_{\mathrm{p}}^{2}=0.31$, while this was not the case with the unfamiliar sequence, $F(1,46)=1.7$, $p>0.19, \eta_{\mathrm{p}}^{2}=0.04$. The finding that the $T_{1}$ versus $T_{2} T_{3}$ difference was actually larger for the familiar than the unfamiliar sequence, $F(1,46)=7.1, p=0.01, \eta_{\mathrm{p}}^{2}=0.13$, confirmed sequence-specific learning in the middle-aged (Indicator b2). For the young adults, the mean of $T_{2}$ and $T_{3}$ was shorter than $T_{1}$ in the familiar and (even) in the unfamiliar 3-key sequence, $F \mathrm{~s}(1,46)>21.0, p \mathrm{~s}<0.001$, $\eta_{\mathrm{p}}^{2}>0.31$. For this younger group too, sequence-specific learning was confirmed by the finding that the $T_{1}$ versus $T_{2} T_{3}$ difference was larger in the familiar than in the unfamiliar sequence, $\quad F(1,46)=22.0, \quad p<0.001$, $\eta_{\mathrm{p}}^{2}=0.32$ (Indicator b2). A higher order planned comparison (contrasting Age, Familiarity, and $T_{1}$ vs. $T_{2} T_{3}$ ) did not show that sequence-specific learning differed for the two age groups with respect to the 3-key sequences, $F(1$, $46)=2.0, p>0.15, \eta_{\mathrm{p}}^{2}=0.04$ (i.e., Indicator b2 rejects Hypothesis 2).

Indications for the use of motor chunks were found only in young adults in that the RTs of their familiar 3-key sequences showed a quadratic component across $T_{1}-T_{3}$ which was caused by a discontinuity at $T_{2}, F(1$, 46) $=30.6, p<0.001, \eta_{\mathrm{p}}^{2}=0.40$. This quadratic component was significantly larger in the young adults than in the middle-aged, $F(1,46)=16.8, p<0.001, \eta_{\mathrm{p}}^{2}=0.27$, and did not even reach significance for the middle-aged, $F(1$, 46) $=0.1, p>0.20, \eta_{\mathrm{p}}^{2}=0.0$. (i.e., Indicator $\mathrm{c} 2$ supports Hypothesis 3). 
Fig. 2 Response times in the familiar and unfamiliar 6-key sequences of the test phase as a function of key position, age group, and structure (i.e., whether or not the 6-key sequence had been practiced with a pause)

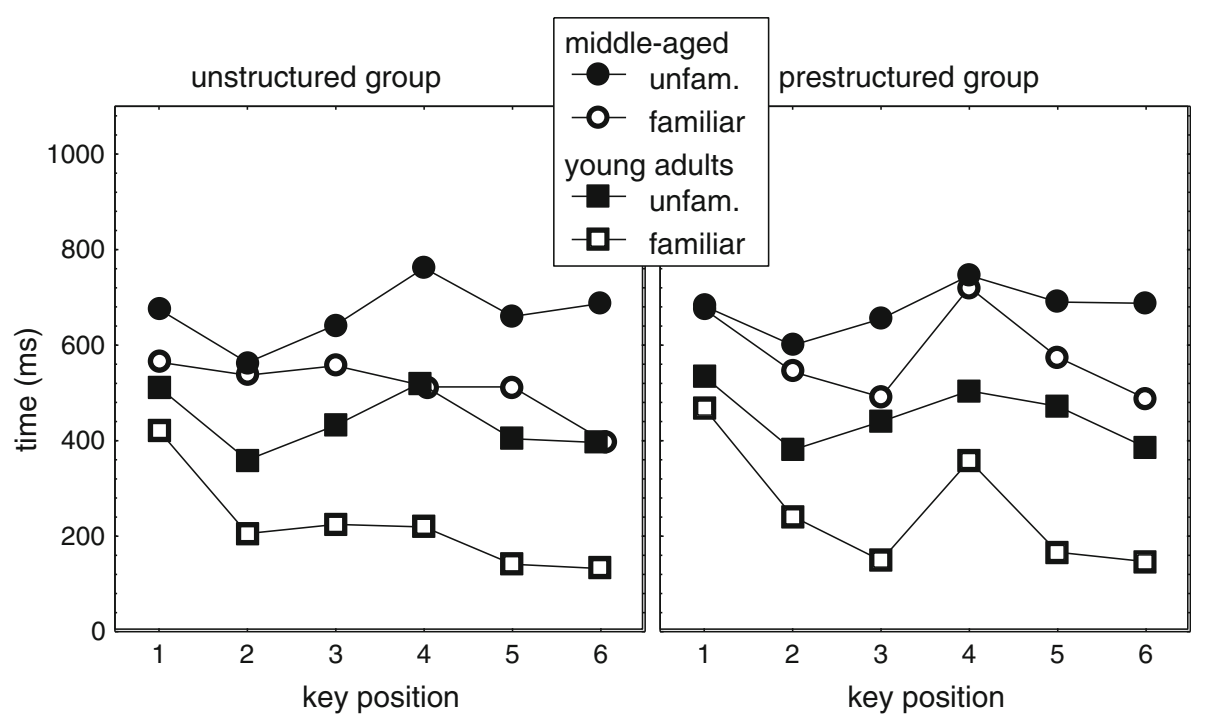

6-Key sequences. Figure 2 suggests use of motor chunks in the familiar 6-key sequences by the prestructured middle-aged participants, and the unstructured and prestructured young adults because of the $T_{1}$ versus $T_{2} T_{3} T_{5} T_{6}$ difference, the discontinuity at $T_{2}$ and in the prestructured 6-key sequence at $T_{5}$, and the relatively long $T_{4}$ in the prestructured sequence. For the unstructured middle-aged participants the RTs seem more in line with associative learning because the advantage of the familiar over the unfamiliar sequence increases gradually with sequential location.

These indications were tested with a mixed $2($ Age $) \times 2$ (Familiarity) $\times 2$ (Structure) $\times 6 \quad$ (Key) ANOVA. It showed main effects of Age, $F(1,44)=105.4, p<0.001$, $\eta_{\mathrm{p}}^{2}=0.71$, Key, $F(5,220)=31.8, p<0.001, \eta_{\mathrm{p}}^{2}=0.42$, and Familiarity, $F(1,44)=151.5, p<0.001, \eta_{\mathrm{p}}^{2}=0.77$. The Familiarity $\times$ Structure $\times$ Key interaction was significant too, $F(5,220)=5.1, p<0.001, \eta_{\mathrm{p}}^{2}=0.10$, while the Age $\times$ Familiarity $\times$ Key interaction was marginally significant, $F(5,220)=2.0, p=0.07, \eta_{\mathrm{p}}^{2}=0.04$. These interactions are in line with the expectation of a $T_{1}$ versus IKI (i.e., mean of $T_{2} T_{3} T_{4} T_{5} T_{6}$ ) difference in familiar and unfamiliar sequences, which differed across age groups and unstructured and prestructured sequences, and that differences between intervals were significant.

Planned comparisons were used to further explore the predictions in the "Introduction". These showed that the advantage of the familiar over the unfamiliar sequence was significant for both age groups separately (Indicator b1 indicating sequence-specific knowledge), $F \mathrm{~s}(1,44)>42.5$, $p$ s $<0.001, \eta_{\mathrm{p}}^{2}=0.49$, but the Familiarity $\times$ Age interaction, $F(1,44)=9.6, p<0.01, \eta_{\mathrm{p}}^{2}=0.18$, suggested that sequence-specific knowledge of the 6-key sequence was less for the middle-aged than for the young adults. Planned comparisons further investigated this interaction and showed that the $T_{1}$ versus IKI difference was significant for the middle-aged in their familiar sequences, $F(1$, $44)=14.6, p<0.001, \eta_{\mathrm{p}}^{2}=0.25$, and not in their unfamiliar sequence, $F(1,44)=1.1, p>0.20, \eta_{\mathrm{p}}^{2}=0.02$. This $T_{1}$ versus IKI difference was larger in the familiar than in the unfamiliar sequences, $F(1,44)=8.1, p<0.01$, $\eta_{\mathrm{p}}^{2}=0.16$. For the young adults, this $T_{1}$ versus IKI difference was significant in both the familiar and unfamiliar sequences, $F \mathrm{~s}(1,44)>14.9, p \mathrm{~s}<0.001, \eta_{\mathrm{p}}^{2}=0.25$, but it was again larger in the familiar sequence, $F(1,44)=31.9$, $p<0.001, \eta_{\mathrm{p}}^{2}=0.42$ (Indicator $\mathrm{b} 2$ indicating sequencespecific learning). This time, the $T_{1}$ versus IKI difference in familiar, relative to unfamiliar, sequences was larger for young adults than for the middle-aged, $F(1,44)=3.9$, $p=0.05, \eta_{\mathrm{p}}^{2}=0.08$, confirming less sequence-specific learning in the middle-aged (i.e., Indicator b2 supports Hypothesis 2).

In line with motor chunk use, young adults of the unstructured group showed a discontinuity at $T_{2}$ by way of a quadratic change across $T_{1}-T_{3}$ of the familiar 6-key sequence, $F(1,44)=14.4, p<0.001, \eta_{\mathrm{p}}^{2}=0.25$. This discontinuity was far from significant in the unstructured middle-aged, $F(1,44)=0.6, p>0.20, \eta_{\mathrm{p}}^{2}=0.01$, and was significantly larger in the unstructured young adults than in the unstructured middle-aged group, $F(1,44)=4.6$, $p<0.05, \eta_{\mathrm{p}}^{2}=0.09$. Likewise, the prestructured young adults showed a discontinuity across $T_{1}-T_{3}$ of the familiar 6-key sequence, $F(1,44)=4.7, p<0.05$, while the prestructured middle-aged did not show this discontinuity, $F(1,44)=1.6, \eta_{\mathrm{p}}^{2}=0.04$. In the prestructured group, however, the age group difference did not reach not significance, $F(1,44)=0.4, p>0.20, \eta_{\mathrm{p}}^{2}=0.01$. So, like with the 3-key sequence the discontinuity criterion at $\mathrm{T}_{2}$ 
confirmed the use of motor chunks in the (unstructured and prestructured) young adults, and not in the (unstructured and prestructured) middle-aged (i.e., Indicator c2 supports Hypothesis 3).

Another criterion for motor chunk use consisted of a relatively long $T_{4}$ at the former pause position in the prestructured 6-key sequence (Indicator c3). In both prestructured age groups $T_{4}$ appeared significantly longer than the mean of $T_{2} T_{3} T_{5} T_{6}, \quad F \mathrm{~s}(1,44)>26.7, \quad p \mathrm{~s}<0.01$, $\eta_{\mathrm{p}}^{2} \mathrm{~s}>0.38$. (Indicator $\mathrm{c} 3$ supports Hypothesis 3 ). This interval difference was not different for the two age groups, $F(1,44)=0.1, p>0.20, \eta_{\mathrm{p}}^{2}=0.00$. Also, for each group this $T_{4}$ versus $T_{2} T_{3} T_{5} T_{6}$ difference was larger in the familiar than in the unfamiliar sequence, $F_{\mathrm{S}}(1,44)>4.1$, $p \mathrm{~s}<0.05, \eta_{\mathrm{p}}^{2}=0.09$, and larger in the prestructured than in the unstructured group, $F \mathrm{~s}(1,44)>4.2, p \mathrm{~s}<0.05$, $\eta_{\mathrm{p}}^{2}=0.09$.

The long $T_{4}$ in the prestructured 6-key sequence of both age groups suggests that the prestructured sequence included two clearly separated motor chunks. This allowed a test of the discontinuity criterion for motor chunking (c2) in the prestructured 6-key sequence across $T_{4}-T_{6}$ too. The corresponding quadratic component was significant in young adults, $F(1,44)=5.5, p<0.05, \eta_{\mathrm{p}}^{2}=0.11$ (Indicator $\mathrm{c} 2$ supports chunking), but not in the middle-aged, $F(1$, $44)<0.7, p s>0.20, \eta_{\mathrm{p}}^{2}=0.02$. Here the group difference did not reach significance either, $F(1,44)=1.2, p>0.20$, $\eta_{\mathrm{p}}^{2}=0.03$.

To increase the power of the comparison of discontinuities at the second response of each 3-key segment, we then tested the discontinuity in both age groups across all three presumed 3-key motor chunks in the young and middle-aged prestructured groups (i.e., one in the 3-key sequence and two in the prestructured 6-key sequence). This showed that the quadratic component across these three key chunks was significant for the young adults, $F(1,44)=19.6, p<0.001, \eta_{\mathrm{p}}^{2}=0.31$, not significant for the middle-aged, $F(1,44)=1.1, p>0.20, \eta_{\mathrm{p}}^{2}=0.02$, and this time it was significantly larger in the young adults than the middle-aged, $F(1,44)=5.6, p<0.05, \eta_{\mathrm{p}}^{2}=0.11$ (i.e., Indicator c2 supports Hypothesis 3).

Finally, we examined the indication for associative learning in the unstructured middle-aged group that was suggested by the advantage of the familiar over the unfamiliar sequence that increased with sequential position (Fig. 2). This indication for associative learning was confirmed by a Familiarity $\times$ Key planned interaction across $T_{2}-T_{6}, F(4,176)=5.9, p<0.001, \eta_{\mathrm{p}}^{2}=0.12$. Indeed, for this group the difference between the unstructured familiar and unfamiliar sequences was larger at $T_{6}$ than at $T_{2}, F(1$, $44)=15.6, p<0.001, \eta_{\mathrm{p}}^{2}=0.26$. In fact, the execution rate of the familiar sequence increased across $T_{2}-T_{6}, F(4$, $176)=4.3, p<0.01, \eta_{\mathrm{p}}^{2}=0.09$, while it actually reduced for the unfamiliar sequence, $F(4,176)=5.5, p<0.001$, $\eta_{\mathrm{p}}^{2}=0.11^{4}$ (Indicator $\mathrm{d} 3$ supports Hypothesis 3$)^{5}$

Interestingly, the apparent use of motor chunks in the prestructured sequence did not lead to the prestructured sequence being executed faster than the unstructured sequence, neither in the middle-aged nor in the young adult group, $F \mathrm{~s}(1,44)<2.1$, $p \mathrm{~s}>0.13, \eta_{\mathrm{p}}^{2}=0.04$ (i.e., Indicator $\mathrm{d} 2$ does not show an advantage of chunk use). The relatively long $T_{4}$ in the prestructured 6-key sequence must have undone the execution rate advantage of that sequence.

Errors. Arcsine transformed error proportions of 3- and 6-key sequences were submitted to mixed 2 (Age) $\times 2$ (Familiarity) $\times 3$ (Key) and 2 (Age) $\times 2$ (Familiarity) $\times 2$ (Structure) $\times 6$ (Key) ANOVAs. Mean error percentages were $1.7 \%$ per key for the 3-key sequence, and $2.3 \%$ per key for the 6-key sequence. The only significant effect in the ANOVA on the 3-key sequences concerned a main effect of Key, $F(2,88)=2.9, p<0.01, \eta_{\mathrm{p}}^{2}=0.06$, indicating that error rate was highest for $R_{2}$ (i.e., 1.1, 3.1, $1.0 \%$, resp.). The ANOVA on the 6-key sequences showed only main effects of Structure, $F(1,44)=6.2, p<.05$, $\eta_{\mathrm{p}}^{2}=.12$ (unstructured vs. prestructured: 2.9 vs. $1.6 \%$ ), Familiarity, $F(1,44)=4.6, p<0.05, \eta_{\mathrm{p}}^{2}=0.09$ (familiar vs. unfamiliar; 1.7 vs. $2.7 \%)$, and $\mathrm{Key}, F(5,220)=5.4$, $p<0.001, \quad \eta_{\mathrm{p}}^{2}=0.11 \quad\left(R_{3} 3.8 \%, R_{5} 3.4 \%\right.$, remaining responses $<2.8 \%)$. Age was never significant, $F_{\mathrm{s}}(1$, $44)<1.4, p s>0.20, \eta_{\mathrm{p}}^{2}<0.03$.

In summary, the RT analyses on the test phase data confirm sequence-specific learning for both age groups in that the familiar 3- and 6-key sequences were executed faster than their unfamiliar counterparts (Indicator b1). Yet, in the 6-key sequence the benefit was smaller for the

\footnotetext{
${ }^{4}$ Performing the same ANOVAs for 3- and 6-key sequences, separately for the three groups for which the test conditions had a particular order showed the same general patterns of significant effects as across all participants (though not always for the higher order interactions) and no significant interactions with test condition order. This rejects the possibility that trial-and-error learning in the single-stimulus condition (not included in this ANOVA) influenced ensuing test conditions.

${ }^{5}$ Visual inspection of the individual RT data suggested that perhaps 4 or 5 of the 12 middle-aged participants may have used motor chunks, but the indicative slow response(s) occurred at different locations and cancelled each other out (cf. Verwey \& Eikelboom, 2003). Problematic with this interpretation is that these effects may have been execution and not motor chunk related in that the slow responses may have been caused also by a particular participant using a slower finger at that location. Also, a chunking interpretation can not explain the gradually increasing advantage of the familiar over the unfamiliar sequences across the entire middle-aged group, and even with different chunking patterns one would expect $R_{2}$ and $R_{6}$ to be relatively fast because 1-key chunks are less likely (cf. Verwey, 2003b). We therefore think the associative learning interpretation is still the best explanation for the middle-aged group as a whole though we acknowledge that some middle-aged probably used motor chunks too.
} 
middle-aged than for the young adults (supporting Hypothesis 2). Likewise, the $T_{1}$ over IKI advantage in the familiar (relative to unfamiliar) sequences showed less sequence-specific learning for the middle-aged participants than the young adults in the 6-key sequence (Indicator b2 supporting Hypothesis 2). Young adults showed evidence for motor chunk use by way of a long $T_{4}$ (Indicator c3) and discontinuities at $R_{2}$ (in 3- and 6-key sequences) and at $R_{5}$ (in the prestructured 6-key sequence) (Indicator c2). The middle-aged only had a relatively long $T_{4}$ in the prestructured 6-key sequence and no significant discontinuities at $R_{2}$ and $R_{5}$. (c3 supported Hypothesis 3, c2 did not). The middle-aged unstructured participants did show a clear indication for associative learning in their 6-key sequence in that the benefit of familiar over unfamiliar sequences gradually increased with sequential position ( $\mathrm{d} 3$ supported Hypothesis 3), though individual data suggest that some middle-aged may have used motor chunks too. The middleaged and young adults did not execute the prestructured 6-key sequence faster than the unstructured 6-key sequence (d2 rejects an advantage of motor chunks).

\section{The single-stimulus condition}

Figure 3 confirmed our expectation that the middle-aged executed fewer familiar sequences correctly than the young adults in the single-stimulus condition. A Kruskal-Wallis ANOVA on the total numbers of correctly executed 3- and 6-key sequences in the single-stimulus condition showed that both these numbers (relative to those in the familiar sequences) were lower across the middle-aged than across the young adults, $H \mathrm{~s}(1, N=48)>15.4, \quad p s<0.001$ (Indicator $\mathrm{c} 1$ confirms Hypothesis 3 ). The ability to execute the familiar sequences in the single-stimulus condition was not significantly different for prestructured and unstructured participants, $H \mathrm{~s}(1, N=48)<0.8, p \mathrm{~s}>0.20$.

In order to analyze the RTs across the participants who in the single-stimulus condition did execute the 3- or 6-key sequences at least once, 2 (Age) $\times 3$ (Sequence: Familiar, Unfamiliar, Single-stimulus) $\times 3 / 6$ (Key) ANOVAs were carried out on the RTs. For the 19 (of 24) middle-aged who had executed the familiar 3-key sequence in the singlestimulus condition the RTs were significantly longer than in the familiar and also than in the unfamiliar sequence conditions (mean RTs 1,148 vs. 532 and $626 \mathrm{~ms}$, resp.), $F_{\mathrm{s}}(1,39)>29.8, p \mathrm{~s}<0.001, \eta_{\mathrm{p}}^{2}>0.43$. All 24 young adults executed the familiar 3-key sequence in the singlestimulus condition, and for them execution rate was lower than in the familiar condition (mean RTs 418 vs. $251 \mathrm{~ms}$ ), $F(1,39)=3.9, p=0.05, \eta_{\mathrm{p}}^{2}=0.09$, but not slower than in the unfamiliar sequence $(400 \mathrm{~ms}), \quad F(1,39)=0.1$, $p>0.20, \eta_{\mathrm{p}}^{2}=0.00$. This slowing of the 3 -key familiar sequence in the single-stimulus relative to the familiar condition was significantly larger for the middle-aged than for the young adults, $F(1,39)=12.6, p<0.01, \eta_{\mathrm{p}}^{2}=0.24$ (suggesting that the middle-aged relied more on an inefficient sequence representation like explicit knowledge, and less on motor chunks).

The corresponding 6-key sequence ANOVA showed that the familiar 6-key sequence was executed more slowly in the single-stimulus condition than in the familiar condition and this time also slower than the unfamiliar sequence condition by the 11 (of 24) middle-aged who had executed the familiar 6-key sequence in the single-stimulus condition (mean RTs 1,177 vs. 532 and $668 \mathrm{~ms}), F(1$, $30)>31.8$, $p \mathrm{~s}<0.001, \eta_{\mathrm{p}}^{2}=0.51$. The 22 young adults who carried out the familiar 6-key sequence in the singlestimulus condition executed it slower without than with
Fig. 3 The mean proportions of correctly completed sequences across participants of each age group in the familiar, singlestimulus, and unfamiliar conditions of the test phase as a function of sequence length

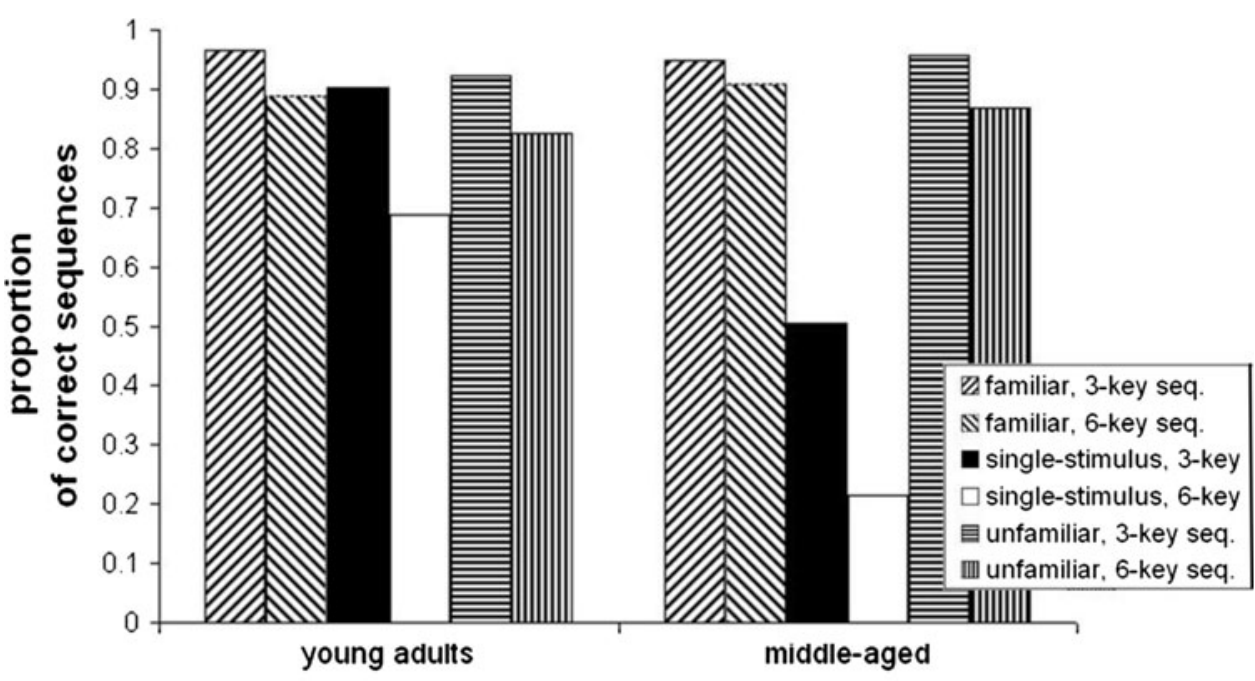

age group 
key-specific stimuli (380 vs. $225 \mathrm{~ms}$ ), $F(1,30)=8.7$, $p<0.001, \eta_{\mathrm{p}}^{2}=0.22$, but not slower than the unfamiliar sequence, $445 \mathrm{~ms}, F(1,30)=1.0, p>0.20, \eta_{\mathrm{p}}^{2}=0.03$. Again, slowing in the single-stimulus condition, relative to the familiar condition, was larger for the middle-aged than for the young adults, $F(1,30)=30.3, p<0.001$, $\eta_{\mathrm{p}}^{2}=0.50$. (Indicator $\mathrm{c} 1$ supports Hypothesis 3 ). The prestructured middle-aged were not faster than the unstructured middle-aged, $F(1,30)=0.35, p>0.20, \eta_{\mathrm{p}}^{2}=0.01$ (rejecting the notion that motor chunks would be more useful here than other forms of sequence knowledge). Yet, it is interesting to see that in both middle-aged groups the increase in RT was caused primarily by a slow $T_{2}-T_{4}$ relative to $T_{5} T_{6}, F \mathrm{~s}(1,30)>12.1, p \mathrm{~s}<0.01, \eta_{\mathrm{p}}^{2}=0.29$, as if later responses were triggered once the first responses had been executed in a more cumbersome and slow way.

In short, the middle-aged executed fewer familiar 3- and 6-key sequences in the single-stimulus condition than the young adults (Indicator c1 supports Hypothesis 3), but this was not higher in the prestructured middle-aged. Those middle-aged who managed to execute the familiar sequences on basis of just $S_{1}$ did so more slowly than the young adults (relative to their own familiar and unfamiliar conditions), suggesting that the middle-aged were more reliant external guidance than the young adults (but not necessarily due to the use of motor chunks as sequence structure had no effect).

\section{Awareness}

Fewer middle-aged than young adults were able to correctly write down their familiar sequences in the recall test (see 'total' columns in Table 1), $\chi^{2} \mathrm{~s}(1)>12.8, p \mathrm{~s}<0.001$ (Indicator e1). Likewise, fewer middle-aged than young participants selected the proper 3-key and 6-key sequences in the subsequent recognition task (familiar 3-key sequence: 12 of the 24 middle-aged vs. 23 of the 24 young adults, i.e. $50 \%$ vs. $96 \%, \chi^{2}(1)=12.8, p<0.001$; 6-key sequence: 10 of 24 middle-aged vs. 23 of 24 , young adults, i.e., 42 vs. $96 \%$,

Table 1 The numbers (and percentages) of middle-aged and young participants who recalled their familiar 3- and 6-key sequences in the awareness test in terms of correctly writing down their familiar sequences. For each group, the participants are further divided into $\chi^{2}(1)=16.4, p<0.001$ ) (Indicator e2). So, the middle-aged were clearly less aware of their familiar sequences than the young adults (supporting Hypothesis 4).

\section{Awareness and the single-stimulus condition}

Table 1 indicates a distinction among three groups of participants. These differed with respect to the use they made of explicit and implicit knowledge of the 3-key and of the 6-key sequences: the no-recall/no-execution (with 5 and 15 participants, resp.), the no-recall/execution (13 and 15 participants), and the recall/execution groups (30 and 18 participants). The fourth (recall/no-execution) group did not include participants. This distribution of participants across the four combinations of recall and execution was unequal for both 3-key and 6-key sequences, $\chi^{2} s(1)>10.8, p s<0.01$. This interaction was significant because participants recalling their sequences were always able to also execute them in the single-stimulus condition, while participants without sequence recall were not always able to execute their sequences (supporting Hypothesis 4). This indicates that explicit knowledge could be used for executing familiar sequences. It is confirmed by the finding that the number of correctly executed familiar sequences in the single-stimulus condition per participant correlated with both their recall and recognition scores, $r \mathrm{~s}(n=48)>0.67, p \mathrm{~s}<0.05$ (supporting Hypothesis 4).

Within the no-recall group there were fewer middleaged participants than young adults who were able to execute their 6-key sequences in the single-stimulus condition ( 8 of $21=38 \%$ vs. 7 of $9=78 \%$ ), $\chi^{2}(1)=4.0$, $p<0.05$. This suggests that the middle-aged without awareness were also less able to use other, implicit sequence knowledge for executing the familiar sequences (like motor chunks or associations).

The earlier test phase ANOVAs on RTs in the 3- and 6-key familiar and unfamiliar sequences were extended with a Recall variable which indicated whether a

those who executed their familiar sequence in the single-stimulus condition at least once versus those who were not able to execute their familiar sequences correctly on basis of just $S_{1}$

\begin{tabular}{|c|c|c|c|c|c|c|}
\hline & \multicolumn{3}{|l|}{ No-recall } & \multicolumn{3}{|l|}{ Recall } \\
\hline & No-execution & Execution & Total & No-execution & Execution & Total \\
\hline \multicolumn{7}{|c|}{ Middle-aged } \\
\hline 3-key & $5(21 \%)$ & $10(42 \%)$ & $15(63 \%)$ & $0(0 \%)$ & $9(38 \%)$ & $9(38 \%)$ \\
\hline 6-key & $13(54 \%)$ & $8(33 \%)$ & $21(87 \%)$ & $0(0 \%)$ & $3(13 \%)$ & $3(13 \%)$ \\
\hline \multicolumn{7}{|c|}{ Young adults } \\
\hline 3-key & $0(0 \%)$ & $3(13 \%)$ & $3(13 \%)$ & $0(0 \%)$ & $21(88 \%)$ & $21(88 \%)$ \\
\hline 6-key & $2(8 \%)$ & 7 (29\%) & $9(37 \%)$ & $0(0 \%)$ & $15(63 \%)$ & $15(63 \%)$ \\
\hline
\end{tabular}


participant had been able to write down their familiar sequences. The resulting mixed 2 (Recall) $\times 2($ Age $) \times 2$ (Familiarity) $\times 3 / 6$ (Key) ANOVAs on RTs in the familiar and unfamiliar sequences showed that across both age groups, participants were generally faster executing familiar and unfamiliar sequences when they were aware of these sequences, 3-key: $94 \mathrm{~ms}, F(1,44)=9.0, p<0.05$, $\eta_{\mathrm{p}}^{2}=0.17, \quad 6$-key: $70 \mathrm{~ms}, \quad F(1,44)=4.7, \quad p<0.05$, $\eta_{\mathrm{p}}^{2}=0.05$. This may only indicate that aware participants are always faster. Yet, this execution rate advantage of aware participants was larger for familiar than for unfamiliar sequences, 3-key: 137 versus $53 \mathrm{~ms}, F(1,44)=3.4$, $p=0.07, \quad \eta_{\mathrm{p}}^{2}=0.07 ;$ 6-key: 119 versus $21 \mathrm{~ms}, \quad F(1$, $44)=8.7, p<0.01, \eta_{\mathrm{p}}^{2}=0.17$. This indicates that even when key-specific stimuli were displayed explicit knowledge was used to execute familiar sequences. This effects reached significance for the middle-aged, $F(1,44)=8.1$, $p<0.01, \eta_{\mathrm{p}}^{2}=0.16$, but not for the young adults, $F(1$, $44)=1.1, p>0.20, \eta_{\mathrm{p}}^{2}=0.02$. (supporting Hypothesis 4 ). The larger execution rate benefit of aware participants in familiar (relative to unfamiliar) sequences was stronger in the middle-aged, $F(1,44)=3.1, p<0.05$ (one-tailed), $\eta_{\mathrm{p}}^{2}=0.07$. In other words, middle-aged with awareness seem to have relied more for executing their familiar sequences on explicit knowledge than young adults with awareness (who were fast any way).

In short, these analyses indicate that awareness (as indicated by recall and recognition scores) was lower in the middle-aged than in the young adults (supporting Hypothesis 4). Participants explicitly recalling their familiar sequences were also always able to execute their sequences in the single-stimulus condition, while only some of the participants without proper recall executed their familiar sequences in response to just $S_{1}$ (supporting Hypothesis 4). Unaware middle-aged were less able than unaware young adults to execute their familiar sequences in the single-stimulus condition, suggesting unaware middle-aged were less able than unaware young adults to use implicit sequence knowledge. While aware young adults did not execute their sequences in the familiar condition faster than unaware young adults, the aware middle-aged appeared to be faster than the unaware middle-aged when executing their sequences in the familiar condition. This suggests that aware middle-aged participants made more use of their explicit sequence knowledge in executing familiar sequences than aware young adults (supporting Hypothesis 4).

Finally, for the middle-aged the ratings on physical health and computer experience correlated significantly with the $T_{1}$ versus $T_{2} T_{3}$ difference in the familiar 3-key sequence, $r s(n=24)>0.47, p s<0.05$. This was neither found with 6-key sequences, nor were there any other correlations with performance or awareness measure.

\section{Discussion}

The aim of the present study was to compare the capacity of middle-aged people to learn sequential motor skills with that capacity of young adults. Using a DSP task we explored the contribution to a familiar keying sequence of motor chunks, and associative and explicit sequence learning. In brief, the results show that despite a similar allover improvement in the practice phase for middle-aged and young adults sequence-specific learning was less in middle-aged participants. Detailed analyses suggested that this was caused by a less pronounced use of motor chunks by the middle-aged in sequences including 3-key segments, and replacing to a large extent the use of motor chunks by associative learning in the unstructured 6-keys sequence. Together with more limited explicit sequence knowledge in the middle-aged this led to higher dependence on keyspecific stimuli in the middle-aged participants.

At first sight the results seemed to corroborate the notion that middle-aged participants and young adults have a similar learning ability in that during practice total sequence execution time reduced to a similar extent (Daselaar et al., 2003; Howard \& Howard, 1992; VoelckerRehage, 2008). However, in line with long existing doubts about the interpretation of learning curves (e.g., Bahrick, Fitts, \& Briggs, 1957; Brown \& Heathcote, 2003), and with more limited sequence-specific learning in elderly (Verwey, 2010), analyses of the present test phase showed that despite the apparent similarity in amount of learning the middle-aged had developed less sequence-specific knowledge of the 6-key sequence than the young adults. In fact, reduced learning in the practice phase by the middleaged was corroborated by an analysis on standardized RTs (Faust et al., 1999). The fact that learning curves in terms of a reduction in absolute RTs were still similar for the two age groups can be reconciled by the notion that the middleaged developed less sequence-specific knowledge for the 6-key sequence than the young adults but overall improvement was comparable because sequence-unspecific task knowledge did not develop as fast as in young adults either (cf. MacKay, 1982; Newell \& Rosenbloom, 1981).

Detailed analyses indicate that the limited sequencespecific learning by the middle-aged was caused by a restricted development of motor chunks in the 3-key and prestructured 6-key sequences. This was suggested by the slower RT decrease after the first response of each 3-key segment (preventing a significant discontinuity) and a higher dependence on key-specific stimuli. In addition, the unstructured 6-key sequence showed significant sequencespecific learning for the middle-aged, but without any signs for motor chunk use. This observation can be attributed to associative learning, which-from the perspective of building up of activation over time-nicely fits the gradual 
decline of response times over key positions. Such learning is in line also with (a) the notion that older people tend to be more dependent on guidance by external stimuli (e.g., Hedel \& Dietz, 2004; Hultsch et al., 1987), (b) similar findings in the DSP task with elderly (Verwey, 2010), ${ }^{6}$ and (c) findings in the serial RT task in which participants of all ages continue responding to key-specific stimuli (e.g., Abrahamse et al., 2010; Keele et al., 2003). The results observed with the unstructured 6-key sequence are hard to explain by the middle-aged dividing (chunking) the 6-key sequence in different ways as that would not explain the gradual advantage of familiar over unfamiliar sequences.

Despite these indications for associative learning of the unstructured 6-keys sequence, the middle-aged of the unstructured group did not execute their 6-key sequence as a whole more slowly than the prestructured middle-aged (rejecting d2). This seems to imply that associative learning is as useful as the use of motor chunks. For the middleaged, the advantage of motor chunks seems to lie especially in the reduced dependence on external guidance rather than in a larger overall execution rate.

These results lead to the conclusion that the middle-aged were less able to use motor chunks. ${ }^{7}$ This seems to have been responsible in the present task for the finding that the middle-aged maintaining their reliance on external guidance during practice (e.g., Hedel \& Dietz, 2004; Hultsch et al., 1987). The finding that the middle-aged had less explicit sequence knowledge than the young adults implies that they were also less able than young adults to compensate for limited motor chunk use by translating explicit sequence knowledge into actual key presses. Research with the serial RT and DSP tasks has indeed demonstrated that older people are less able to develop explicit sequence knowledge (see Gaillard et al., 2009; Prull et al., 2000; Verwey, 2010). This is sensible if we consider that the development of explicit knowledge presumably involves testing hypotheses about response order (e.g., Cleeremans \& Sarrazin, 2007). During sequence execution the middleaged may have been less able to switch between

\footnotetext{
${ }^{6}$ In line with the notion that associative learning of simple sequences is not affected much by age (Howard, et al., 2004), a cross-experiment ANOVA showed no larger benefits in the unstructured 6-key sequences (relative to the unfamiliar sequence) for the present middle-aged than for the elderly in Verwey $(2010), F(1,44)=0.0$, $p>0.20, \eta_{\mathrm{p}}^{2}<.0 .0$. That is, for the middle-aged the benefit of the unstructured familiar over the unfamiliar 6-key sequence increased by $162 \mathrm{~ms}$ from $R_{2} R_{3}$ to $R_{5} R_{6}$ [i.e., from $56 \mathrm{~ms}$ across $R_{2}$ and $R_{3}$ to $218 \mathrm{~ms}$ across $R_{5}$ and $R_{6}, F(1,44)=4.2, p<0.05, \eta_{\mathrm{p}}^{2}<0.09$ ], while for the elderly in Verwey (2010) it had increased by $172 \mathrm{~ms}$ [i.e., from $96 \mathrm{~ms}$ across $R_{2}$ and $R_{3}$ to $268 \mathrm{~ms}$ across $R_{5}$ and $R_{6}, F(1$, 44) $\left.=4.8, p<0.05, \eta_{\mathrm{p}}^{2}<0.10\right]$.

${ }^{7}$ It has to be acknowledged that we used the RT pattern as indicator for the underlying learning mechanism. In theory, however, some unknown control mechanism might also be responsible.
}

responding and hypotheses testing. It thus seems that the middle-aged performed poorly in the single-stimulus condition because motor chunk development was limited (especially in the unstructured 6-key sequence), and because they lacked the flexibility that is characteristic of explicit sequence knowledge. Moreover, the latter two may be highly related to each other. Hence, it has been suggested that the formation and utilization of motor chunks requires explicit sequence knowledge (Hoffmann \& Koch, 1997; Koch, 2007; Verwey, 2003a). From this perspective, the lack of chunking observed in the middle-aged can be attributed directly to their limited explicit sequence knowledge.

There are more explanations the middle-aged may have made less use of motor chunks (cf. Verwey, 2010). First, aging may reduce the capacity to develop motor chunks, for example because associations within motor chunks develop more poorly and result in weaker associations (Mitchell, Johnson, Raye, Mather, \& D'Esposito, 2000; Naveh-Benjamin, 2000). These associations may have prevented the use of motor chunks because the associations were too weak to support responding without looking at the key-specific stimuli, while they still primed responding to these stimuli (cf. Cleeremans \& Jiménez, 2002). Limited development of associations may be caused by a degeneration of brain structures like the frontal lobe or the basal ganglia (which are assumed to play an important role in motor chunking, e.g., Verwey et al., 2002). Indeed, with age a dopamine receptor deficiency seems to develop in the basal ganglia (Baeckman, Ginovart, Dixon et al., 2000; Braver, Barch, Keys et al., 2001). In addition, associations may have been weak because cognitive slowing induces long interkey intervals (Curran, 1997; Dennis, Howard, \& Howard, 2006; MacKay, 1982).

Second, motor chunks may not have developed easily in the middle-aged because they did not start off preparing and executing successive responses in an integrated way (Klapp, 1995; Sternberg, Monsell, Knoll, \& Wright, 1978), This may have been caused by their more limited working memory (WM) capacity (e.g., Reuter-Lorenz et al., 2000). Bo and Seidler (2009), for example, observed that WM capacity correlates with the organization in chunks of motor sequences. Hence, the 3-key and prestructured 6-key sequences still showed the signs of a chunking process in the middle-aged as the highly salient series of 3 elements did not exceed WM capacity. The unstructured 6-key sequence, however, did not provide the aid of predetermined segmentation, and thereby fell victim to declined WM capacity in the middle-aged (cf. Bo et al., 2009). It must be noted that a WM explanation of the current results may also be related to the claim that motor chunking requires explicit sequence knowledge, as it is widely acknowledged that the constructs of WM and awareness 
are related to one another (e.g., Shah \& Miyake, 1999). While in the present experiment the use of motor chunks seems not to critically depend on awareness in young adults, Table 1 suggests that awareness of the sequence may be more important for the (perhaps more cautious) middle-aged to execute their familiar sequences. Finally, the middle-aged may not have prepared sequences as a whole due to reduced planning abilities (e.g., de Jong, 2001).

Third, it is possible that the middle-aged did develop motor chunks, but did not use them because their reduced cognitive flexibility prevented them from fully switching from reaction to sequencing mode, or because they wanted to prevent errors. Explicit instruction to ignore key-specific stimuli as soon as possible might be useful in that case. Fourth, when young adults execute longer keying sequences they probably develop a series of motor chunks. These motor chunks need to be concatenated by some higher level process (e.g., Verwey et al., 2002, 2009). The middle-age may have had difficulty concatenating successive motor chunks in the unstructured 6-key sequence because $S_{4}$ was not emphasized by the occurrence of a pause.

In conclusion, the results indicate that in absolute terms the middle-aged improved as much as the young adults (supporting Hypothesis 1b) though they were actually slower (supporting Hypothesis 1a); Their limited development of sequence-specific knowledge of 6-key sequences (supporting Hypothesis 2) was caused by reduced use of both motor chunks and explicit sequence knowledge (supporting Hypotheses 3 and 4). We cannot be certain that the actual development of motor chunks was less pronounced in the middle-aged, but this seems a reasonable explanation for the indications that they relied less than young adults on the use of motor chunks and more on the key-specific stimuli. Especially in the unstructured 6-key sequence the middle-aged continued responding to keyspecific stimuli and, like the elderly in Verwey (2010), their responses seem to have benefited more from associative learning than using motor chunks. Notice that the present perspective on associative learning suggests that a gradually increasing effect of practice may be observed also at the start of each block in the serial RT task (and perhaps following an error).

The present indications that the skilled execution of discrete keying sequences involves several learning mechanisms like using motor chunks and explicit knowledge, and associative learning (for a further division of sequence knowledge, see De Kleine \& Verwey, 2009) confirm recent findings using a secondary task paradigm (Verwey et al., 2010), and indicate that-like in the serial RT task (Shanks \& St. John, 1994)-performance of familiar DSP sequences is not process-pure. Extensive practice seems to induce a gradual change in the relative contributions of these mechanisms to the execution of movement sequences, and this change is modified by aging in that motor chunks and explicit sequence knowledge are used less by older people. This implies that the slower execution of movement sequences observed with older people is not only caused by slowing in general, but also by a limited reliance on efficient mechanisms like applying motor chunks and explicit knowledge. The present research suggests that older people may benefit when training of sequential motor skills involves (a) practicing movement patterns in short series, (b) preparing and executing short series of movements in an integrated fashion (i.e., as a whole), and (c) an attempt to become aware of the individual movements that make up the movement pattern (cf. Seidler, 2007).

Acknowledgments This study was carried out by students in Willem Verwey's laboratory classes. We thank Carina Snijder, Viola Hamer, and Carola Engbers for running the middle-aged participants in this experiment. This research was supported in part by grant 400-07-097 from the Netherlands Organization for Scientific Research NWO.

Open Access This article is distributed under the terms of the Creative Commons Attribution Noncommercial License which permits any noncommercial use, distribution, and reproduction in any medium, provided the original author(s) and source are credited.

\section{References}

Abrahamse, E. L., Jiménez, L., Verwey, W. B., \& Clegg, B. A. (2010). Representing serial action: A dynamic approach. Psychological Bulletin \& Review, 17, 603-623.

Baars, B. J. (1997). In the theater of consciousness: The workspace of the mind. New York: Oxford University Press.

Baeckman, L., Ginovart, N., Dixon, R. A., Robins Wahlin, T.-B., Wahlin, A., Halldin, C., et al. (2000). Age-related cognitive deficits mediated by changes in the striatal dopamine system. American Journal of Psychiatry, 157, 635-637.

Bahrick, H. D., Fitts, P. M., \& Briggs, C. E. (1957). Learning curves-facts or artifacts? Psychological Bulletin, 54, 256-268.

Bo, J., Borza, V., \& Seidler, R. D. (2009). Age-related declines in visuospatial working memory correlate with deficits in explicit motor sequence learning. Journal of Neurophysiology, 102, 2744-2754.

Bo, J., \& Seidler, R. D. (2009). Visuospatial working memory capacity predicts the organization of acquired explicit motor sequences. Journal of Neurophysiology, 101, 3116-3125.

Bohlanda, J. W., \& Guenther, F. H. (2006). An fMRI investigation of syllable sequence production. Neuroimage, 32, 821-841.

Braver, T. S., Barch, D. M., Keys, B. A., Carter, C. S., Cohen, J. D., Kaye, J. A., et al. (2001). Context processing in older adults: Evidence for a theory relating cognitive control to neurobiology in healthy aging. Journal of Experimental Psychology: General, 130, 746-763.

Brown, S., \& Heathcote, A. (2003). Averaging learning curves across and within participants. Behavior Research Methods, Instruments \& Computers, 35(1), 11-21. 
Cleeremans, A., \& Jiménez, L. (2002). Implicit learning and consciousness: A graded, dynamic perspective. In R. M. French \& A. Cleeremans (Eds.), Implicit learning and consciousness: An empirical, computational and philosophical consensus in the making? (pp. 1-40). Hove: Psychology Press.

Cleeremans, A., \& Sarrazin, J.-C. (2007). Time, action, and consciousness. Human Movement Science, 26, 180-202.

Curran, T. (1997). Effects of age on implicit sequence learning: Accounting for sequence structure and explicit knowledge. Psychological Research, 60, 24-41.

Daselaar, S. M., Rombouts, S. A. R. B., Veltman, D. J., Raaijmakers, J. G. W., \& Jonker, C. (2003). Similar network activated by young and old adults during the acquisition of a motor sequence. Neurobiology of Aging, 24, 1013-1019.

De Jong, R. (2001). Adult age differences in goal activation and goal maintenance. European Journal of Cognitive Psychology, 13, 71-90.

De Kleine, E. L., \& Verwey, W. B. (2009). Representations underlying skill in the Discrete Sequence Production task: Effects of hand used and hand position on the discrete sequence production task. Psychological Research, 73, 685-694.

Dennis, N. A., Howard, J. H., \& Howard, D. V. (2006). Implicit sequence learning without motor sequencing in young and old adults. Experimental Brain Research, 175, 153-164.

Durkin, M., Prescott, L., Furchtgott, E., Cantor, J., \& Powell, D. A. (1995). Performance but not acquisition of skill learning is severely impaired in the elderly. Archives of Gerontology and Geriatrics, 20, 167-183.

Faust, M. E., Balota, D. A., Spieler, D. H., \& Ferraro, F. R. (1999). Individual differences in information processing rate and amount: Implications for group differences in response latency. Psychological Bulletin, 125, 777-799.

Gaillard, V., Destrebecqz, A., Michiels, S., \& Cleeremans, A. (2009). Effects of age and practice in sequence learning: A graded account of ageing, learning, and control. European Journal of Cognitive Psychology, 21, 255-282.

Gallistel, C. R. (1980). The organization of action: a new synthesis. Hillsdale: Erlbaum.

Gottsdanker, R., Perkins, T., \& Aftab, J. (1986). Studying reaction time with nonaging intervals: An effective procedure. Behavior Research Methods, Instruments, and Computers, 18, 287-292.

Hedel, H. J. A., \& Dietz, V. (2004). The influence of age on learning a locomotor task. Clinical Neurophysiology, 115, 2134-2143.

Hoffmann, J., \& Koch, I. (1997). Stimulus-response compatibility and sequential learning in the serial reaction time task. Psychological Research, 60, 87-97.

Howard, D. V., \& Howard, J. H., Jr. (1992). Adult age differences in the rate of learning serial patterns: Evidence from direct and indirect tests. Psychology and Aging, 7, 232-241.

Howard, J. H., Jr., Howard, D. V., Dennis, N. A., Yankovich, H., \& Vaidya, C. J. (2004). Implicit spatial contextual learning in healthy aging. NeuroPsychology, 18, 124-134.

Hultsch, D. F., Hertzog, C., \& Dixon, R. A. (1987). Memory selfknowledge and self-efficacy in the aged. In N. L. Lowe \& C. J. Brainerd (Eds.), Cognitive development in adulthood: Progress in cognitive developmental research (pp. 65-92). New York: Springer .

Jiménez, L. (2008). Taking patterns for chunks: is there any evidence of chunk learning in continuous serial reaction time tasks? Psychological Research, 72, 387-396.

Jiménez, L., Méndez, A., Pasquali, A., Abrahamse, E. L., \& Verwey, W.B. Chunking by colors: Assessing discrete learning in a continuous serial reaction-time task (submitted).

Keele, S. W., Ivry, R. B., Hazeltine, E., Mayr, U., \& Heuer, H. (2003). The cognitive and neural architecture of sequence representations. Psychological Review, 110, 316-339.
Klapp, S. T. (1995). Motor response programming during simple and choice reaction time: The role of practice. Journal of Experimental Psychology: Human Perception and Performance, 21, 1015-1027.

Koch, I. (2007). Anticipatory response control in motor sequence learning: Evidence from stimulus-response compatibility. Human Movement Science, 26, 257-274.

Koch, I., \& Hoffmann, J. (2000). Patterns, chunks, and hierarchies in serial reaction-time tasks. Psychological Research, 63, 22-35.

Li, K., \& Lindenberger, V. (2002). Relations between aging sensory/ sensorimotor and cognitive functions. Neuroscience and Biobehavioral Reviews, 26, 777-783.

MacKay, D. G. (1982). The problems of flexibility, fluency, and speed-accuracy trade-off in skilled behavior. Psychological Review, 89, 483-506.

Mitchell, K. J., Johnson, M. K., Raye, C. L., Mather, M., \& D'Esposito, M. (2000). Aging and reflective processes of working memory: Binding and test load deficits. Psychology and Aging, 15, 527-541.

Naveh-Benjamin, M. (2000). Adult age differences in memory performance tests of an associative deficit hypothesis. Journal of Experimental Psychology. Learning, Memory, and Cognition, $26,1170-1187$.

Newell, A., \& Rosenbloom, P. (1981). Mechanisms of skill acquisition and the law of practice. In J. R. Anderson (Ed.), Cognitive skills and their acquisition (pp. 1-55). Hillsdale: Erlbaum.

Paillard, J. (1960). Neurophysiology. In H.W. Magoun, Handbook of physiology. A critical comprehensive presentation of physiological knowledge and concepts (Section 1, Neurophysiology, vol. III, Chapter 67, The pattern of skilled movements, pp. 16791708). Washington, DC: American Physiological Society.

Panzer, S., Krueger, M., Muehlbauer, T., Kovacs, A., \& Shea, C. H. (2009). Inter-manual transfer and practice: Coding of simple motor sequences. Acta Psychologica, 131, 99-109.

Park, J.-H., \& Shea, C. H. (2005). Sequence learning: response structure and effector transfer. Quarterly Journal of Experimental Psychology, 58A, 387-419.

Park, J.-H., Wilde, H., \& Shea, C. H. (2004). Part-whole practice of movement sequences. Journal of Motor Behavior, 36, 51-61.

Perry, M. E., McDonald, C. R., Hagler, D. J., Jr., Gharapetian, L., Kuperman, J. M., Koyamae, A. K., et al. (2009). White matter tracts associated with set-shifting in healthy aging. NeuroPsychologia, 47, 2835-2842.

Prull, M. W., Gabrieli, J. D., \& Bunge, S. A. (2000). Age-related changes in memory: A cognitive neuroscience perspective. In F. I. Craik \& T. A. Salthouse (Eds.), The handbook of cognitive aging and cognition (2nd ed.) (pp. 91-153). Mahwah: Lawrence Erlbaum Associates, Inc.

Rabbitt, P. (1997). Ageing and human skill: A 40th anniversary. Ergonomics, 40, 962-981.

Reuter-Lorenz, P. A., Jonides, J., Smith, E. E., Hartley, A., Miller, A., Marshuetz, C., et al. (2000). Age differences in the frontal lateralization of verbal and spatial working memory revealed by PET. Journal of Cognitive Neuroscience, 12, 174-187.

Rhodes, B. J., Bullock, D., Verwey, W. B., Averbeck, B. B., \& Page, M. P. A. (2004). Learning and production of movement sequences: Behavioral, neurophysiological and modeling perspectives. Human Movement Science, 23, 699-746.

Rynning, E. (2008). The ageing populations of Europe-Implications for health systems and patients' rights. European Journal of Health Law, 15, 297-306.

Sakai, K., Kitaguchi, K., \& Hikosaka, O. (2003). Chunking during human visuomotor sequence learning. Experimental Brain Research, 152, 229-242.

Salthouse, T. A., McGuthry, K. E., \& Hambrick, D. Z. (1999). A framework for analyzing and interpreting differential aging 
patterns: Application to three measures of implicit learning. Aging, NeuroPsychology, and Cognition, 6, 1-18.

Seidler, R. D. (2007). Older adults can learn to learn new motor skills. Behavioral Brain Research, 183, 118-122.

Seidler, R. D., Bernard, J. A., Burutolu, T. B., Fling, B. W., Gordon, M. T., Gwin, J. T., et al. (2010). Motor control and aging: Links to age-related brain structural, functional, and biochemical effects. Neuroscience and Biobehavioral Reviews, 34, 721-733.

Shah, P., \& Miyake, A. (1999). Models of working memory: An introduction. In A. Miyake \& P. Shah (Eds.), Models of working memory: Mechanisms of active maintenance and executive control (pp. 1-27). Cambridge: Cambridge University Press.

Shanks, D. R., \& St. John, M. F. (1994). Characteristics of dissociable human learning systems. Behavioral and Brain Sciences, 17, 367-447.

Shea, C. H., Park, J.-H., \& Wilde Braden, H. (2006). Age-related effects in sequential motor learning. Physical Therapy, 4, $478-488$

Spiegel, R., \& McLaren, I. P. L. (2006). Associative sequence learning in humans. Journal of Experimental Psychology: Animal Behavior Processes, 32, 150-163.

Sternberg, S., Monsell, S., Knoll, R. L., \& Wright, C. E. (1978). The latency and duration of rapid movement sequences: Comparisons of speech and typewriting. In G. E. Stelmach (Ed.), Information processing in motor control and learning (pp. 117-152). New York: Academic Press.

van Mier, H., \& Hulstijn, W. (1993). The effects of motor complexity and practice on initiation time in writing and drawing. Acta Psychologica, 84, 231-251.

van Mier, H. I., \& Petersen, S. E. (2006). Intermanual transfer effects in sequential tactuomotor learning: Evidence for effector independent coding. Neuropsychologia, 44, 939-949.

Verhaeghen, P., \& Salthouse, T. A. (1997). Meta-analyses of agecognition relations in adulthood: Estimates of linear and nonlinear age effects and structural models. Psychological Bulletin, 122, 231-249.

Verwey, W. B. (1995). A forthcoming key press can be selected while earlier ones are executed. Journal of Motor Behavior, 27, 275-284.
Verwey, W. B. (1996). Buffer loading and chunking in sequential keypressing. Journal of Experimental Psychology: Human Perception and Performance, 22, 544-562.

Verwey, W. B. (1999). Evidence for a multi-stage model of practice in a sequential movement task. Journal of Experimental Psychology: Human Perception and Performance, 25, 1693-1708.

Verwey, W. B. (2001). Concatenating familiar movement sequence: The versatile cognitive processor. Acta Psychologica, 106, 69-95.

Verwey, W. B. (2003a). Processing modes and parallel processors in producing familiar keying sequences. Psychological Research, 67, 106-122.

Verwey, W. B. (2003b). Effect of sequence length on executing familiar keying sequences: Lasting segmentation and preparation? Journal of Motor Behavior, 35, 343-354.

Verwey, W. B. (2010). Diminished motor skill development in elderly: indications for limited motor chunk use. Acta Psychologica, 134, 206-214.

Verwey, W. B., Abrahamse, E. L., \& De Kleine, E. (2010). Cognitive processing in automated discrete keying sequences. Frontiers Psychology, 1(32), 1-13.

Verwey, W. B., Abrahamse, E. L., \& Jiménez, L. (2009). Segmentation of relatively short keying sequences does not transfer to other sequences. Human Movement Science, 28, 348-361.

Verwey, W. B., \& Dronkert, Y. (1996). Practicing a structured continuous keypressing task: Motor chunking or rhythm consolidation? Journal of Motor Behavior, 28, 71-79.

Verwey, W. B., \& Eikelboom, T. (2003). Evidence for lasting sequence segmentation in the discrete sequence production task. Journal of Motor Behavior, 35, 171-181.

Verwey, W. B., Lammens, R., \& van Honk, J. (2002). On the role of the SMA in the discrete sequence production task. A TMS study. Neuropsychologia, 40, 1268-1276.

Voelcker-Rehage, C. (2008). Motor-skill learning in older adults-a review of studies on age-related differences. European Review on Aging and Physical Activity, 5, 5-6.

Wightman, D. G., \& Lintern, G. (1985). Part-task training for tracking and manual control. Human Factors, 27, 179-209.

Winer, B. J., Brown, D. R., \& Michels, K. M. (1991). Statistical principles in experimental design. New York: McGraw-Hill. 